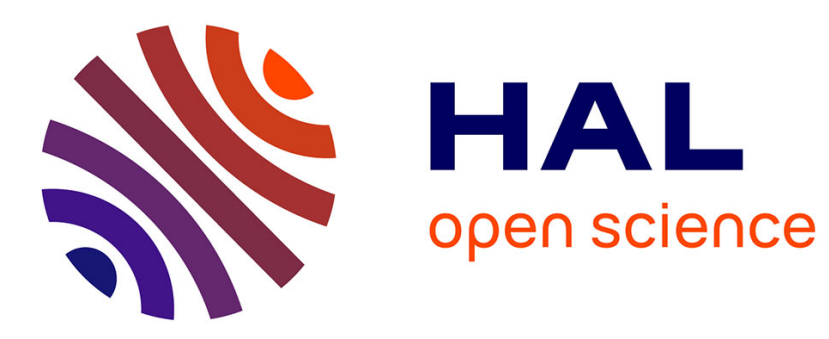

\title{
Travelling waves in a cylindrical magnetohydrodynamically forced flow
}

Jean Boisson, Andrei Klochko, François Daviaud, Sébastien Aumaître

\section{To cite this version:}

Jean Boisson, Andrei Klochko, François Daviaud, Sébastien Aumaître. Travelling waves in a cylindrical magnetohydrodynamically forced flow. Physics of Fluids, 2012, pp.044101. hal-01152717

\section{HAL Id: hal-01152717 https://hal.science/hal-01152717}

Submitted on 19 May 2015

HAL is a multi-disciplinary open access archive for the deposit and dissemination of scientific research documents, whether they are published or not. The documents may come from teaching and research institutions in France or abroad, or from public or private research centers.
L'archive ouverte pluridisciplinaire HAL, est destinée au dépôt et à la diffusion de documents scientifiques de niveau recherche, publiés ou non, émanant des établissements d'enseignement et de recherche français ou étrangers, des laboratoires publics ou privés. 


\section{AIP Fluids}

\section{Travelling waves in a cylindrical magnetohydrodynamically forced flow}

J. Boisson, A. Klochko, F. Daviaud, V. Padilla, and S. Aumaître

Citation: Phys. Fluids 24, 044101 (2012); doi: 10.1063/1.3694804

View online: http://dx.doi.org/10.1063/1.3694804

View Table of Contents: http://pof.aip.org/resource/1/PHFLE6/v24/i4

Published by the American Institute of Physics.

\section{Related Articles}

Two-dimensional numerical analysis of electroconvection in a dielectric liquid subjected to strong unipolar injection

Phys. Fluids 24, 037102 (2012)

Properties of bubbled gases transportation in a bromothymol blue aqueous solution under gradient magnetic fields

J. Appl. Phys. 111, 07B326 (2012)

Magnetohydrodynamic flow of a binary electrolyte in a concentric annulus

Phys. Fluids 24, 037101 (2012)

Optimal transient disturbances behind a circular cylinder in a quasi-two-dimensional magnetohydrodynamic duct flow

Phys. Fluids 24, 024105 (2012)

Stability of a nonlinear magnetic field diffusion wave

Phys. Plasmas 19, 022706 (2012)

\section{Additional information on Phys. Fluids}

Journal Homepage: http://pof.aip.org/

Journal Information: http://pof.aip.org/about/about_the_journal

Top downloads: http://pof.aip.org/features/most_downloaded

Information for Authors: http://pof.aip.org/authors

\section{ADVERTISEMENT}

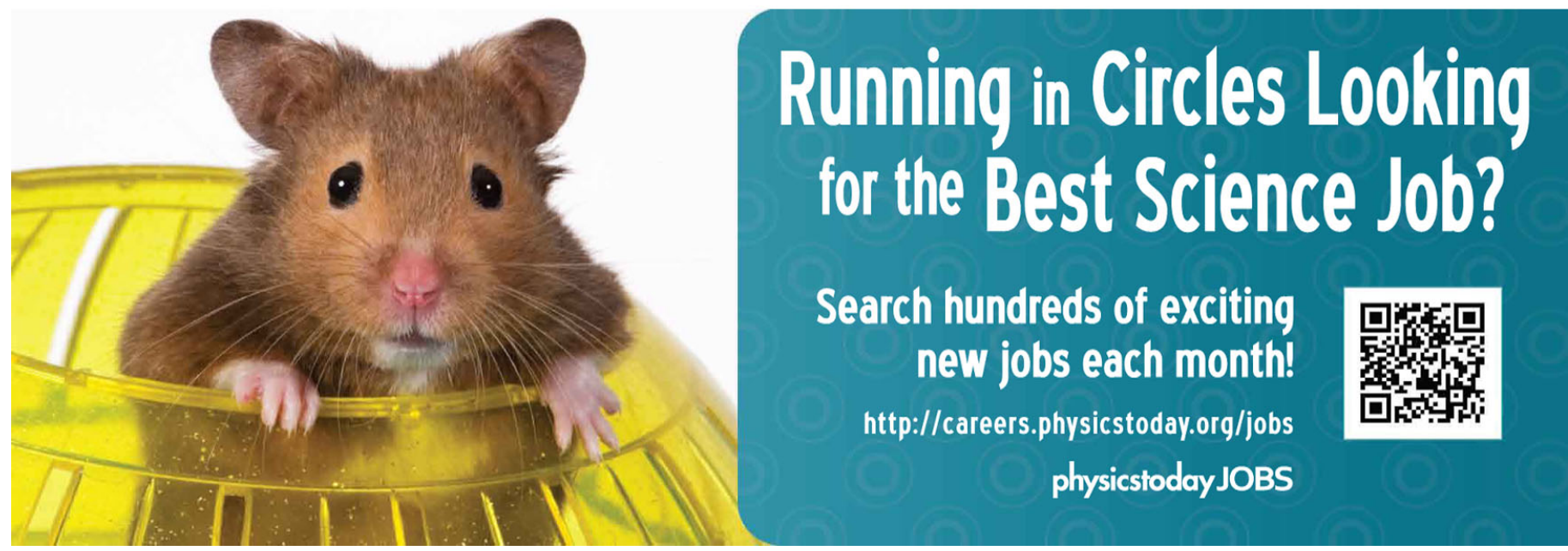




\title{
Travelling waves in a cylindrical magnetohydrodynamically forced flow
}

\author{
J. Boisson, A. Klochko, F. Daviaud, V. Padilla, and S. Aumaître ${ }^{a)}$ \\ GIT-SPEC, DSM, CNRS URA 2464, CEA-Saclay, F-91191 Gif-sur-Yvette, France
}

(Received 11 May 2011; accepted 3 January 2012; published online 4 April 2012)

\begin{abstract}
We present an experimental study of a liquid metal flow electromagnetically forced in a large aspect ratio coaxial cylindrical geometry. An azimuthal Lorentz force is applied on the liquid metal gap, through a radial current and an axial magnetic field. Using ultrasonic velocity measurements, we focus on the effect of these two parameters on the flow properties. We show that, depending on the strength of the magnetic field and not only on the applied Lorentz force, different dynamical states exist. We first observe a stationary structure at low forcing. Then, two other regimes of different travelling waves are exhibited at higher forcing. We characterize them by their different frequencies and speeds. Higher magnetic fields clearly promote the faster waves. Connections with other magnetohydrodynamics instabilities are discussed. (c) 2012 American Institute of Physics. [http://dx.doi.org/10.1063/1.3694804]
\end{abstract}

\section{INTRODUCTION}

Flows of electrically conducting fluids arise in various phenomena ranging from astro- and geophysical to industrial processes ${ }^{1,2}$ and wave phenomena, such as Alfven-wave, ${ }^{3}$ are expected in such fluids although they are not always easy to highlight in lab experiment. In these flows, the Lorentz force $\vec{J} \times \vec{B}$, with $\vec{J}$ being the current density and $\vec{B}$ the magnetic field, plays a significant role. It can either modify and saturate magneto-hydrodynamic (MHD) instability like in fluid dynamo action $^{1,4}$ or drive flows by a suitable arrangement of imposed magnetic fields and currents, like in electromagnetic pumps and magnetic stirring. ${ }^{2}$ Although it is intrinsically a volumic force, the induction phenomena can constrain the electric current in boundary layers letting the fluid bulk free of forcing. The size of the boundary layer, $e_{H}=H / H a$, is controlled by the Hartmann number $H a=B_{o} H \sqrt{\sigma / \rho \nu}$ with $B_{o}$ the imposed magnetic field, $H$ a characteristic length, $\sigma$ the electrical conductivity, $\rho$ the density of the fluide, and $v$ its kinematic viscosity. ${ }^{3}$ Moreover, an anisotropic damping due to the Lorentz force and Joule dissipation tends to reduce fluctuations and to promote a $2 \mathrm{D}$ velocity field by canceling the vortices perpendicular to the imposed magnetic field. ${ }^{2,5}$ One way to study these phenomena is to use a well-defined geometry between two coaxial cylindrical electrodes placed in an axial magnetic field. Without electrical current but with a motion driven by moving boundaries, the stability of this Taylor-Couette flow for conducting fluid with a additional imposed magnetic field, has been studied in Ref. 4. It has been shown that this geometry can generate oscillatory modes. ${ }^{6}$ More recently, similar geometries have been experimentally used to exhibit the magneto-rotational instability which is assumed to play a key role in accretion disks. ${ }^{7,8}$

Instabilities can also be expected when the flow is only driven by a Lorentz force. Indeed, the profile of the tangential velocity $u_{\theta}$ can be computed in an infinite cylinder neglecting the induction effects $^{9}$

$$
u_{\theta}(x)=\frac{B_{o} i_{o} R_{1}\left[\left(x \ln (\eta x)-\eta^{2} x \ln (x)\right)-\frac{\ln (\eta)}{x}\right]}{4 \pi \rho v\left(\eta^{2}-1\right)}
$$

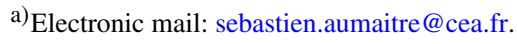


with $i_{o}$ the imposed current by unit of length, $\eta=R_{1} / R_{2}$ the ratio of the radius of the inner electrode, $R_{1}$, over the outer electrode, $R_{2}, x=r / R_{1}$ the radial coordinate normalized by $R_{1}, \rho$ the fluid density, and $v$ the kinematic viscosity. Such a velocity profile is actually unstable following the Rayleigh criterion. ${ }^{4}$ Some instabilities phenomena have been studied in the early 1980s (Ref. 10) and experimental evidence has been reported ${ }^{11}$ for a limited range of forcing in a device with $\Gamma=\left(R_{2}-R_{1}\right) / H \ll 1$ with $H$ the cell height. The same kind of devices, with $\Gamma \sim 1$ have been used to study more specifically turbulent boundary layers problems in this configuration. ${ }^{12,13}$ Because of the difficulty to use usual anemometry techniques in liquid metals, ${ }^{14}$ the velocity was not directly measured all these experiments. Local or global potential measurements were instead performed. However, it has been shown in that measurements of velocity profiles by using ultrasonic techniques are also relevant in liquid metal MHD. ${ }^{15-17}$

In this work, we have applied ultrasonic technique to study velocity profiles of a liquid metal alloy subjected to a Lorentz forcing, between two motionless cylindrical electrodes. We will especially focus on the respective action of the driving current and the magnetic field on the flow. In a first part, we detail the experimental device. In a second part, we first present the behaviours of the mean velocity when the intensity of the input current and the applied magnetic field are changed independently. Then, we show the time evolution of the velocity profile in order to exhibit the two distinct wave regimes that we can observe depending on the magnetic strength, for a given applied Lorentz force. The last part is devoted to discussion and concluding remarks.

\section{EXPERIMENTAL SETUP}

\section{A. Cell description}

Figure 1 shows a vertical cut of the experimental device. An azimuthal Lorentz force is generated by a radial current and an axial magnetic field in order to force a cylindrical layer of liquid alloy: galinstan. ${ }^{18}$ In the design of the experiment, a special care has been devoted to the homogeneity of the input current and magnetic field, without fluid motion. The inner and the outer electrodes are made of a large mass of copper in order to limit the Joule effect within the cell and to allow the current

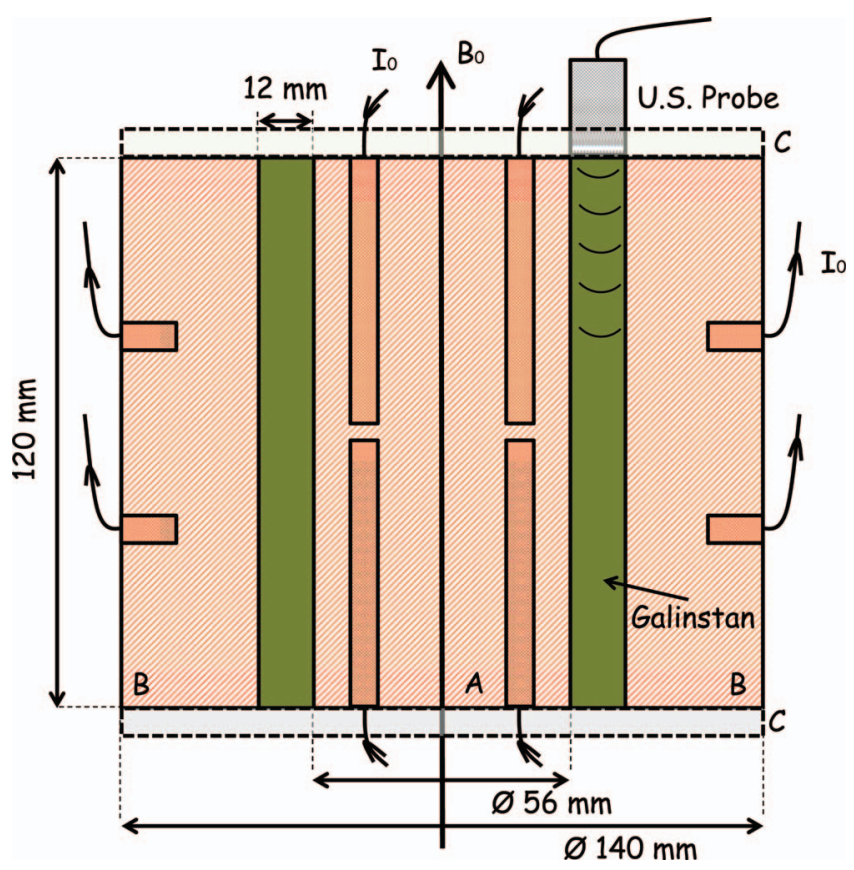

FIG. 1. Schematic view of the experimental setup. A is the inner cylinder (copper) B corresponds to the outer cylinder (copper), $\mathrm{C}$ is the polycarbonate covers. The liquid metal (galinstan) is represented in green. 
homogenization. Indeed we benefit from the conductivity difference between copper and galinstan (the ratio of their electrical conductivity is about 16) to homogenize the current in the bulk of the electrodes before it crosses galinstan. This is also why we divide the current arrivals and departures in eight axisymmetric locations (see Figure 1) in the top and the bottom for the inner cylinder (A), and on the lateral surface for the outer cylinder (B). Moreover the eight current arrivals enter deep in the mass in the inner cylinder. With our filling procedure, the wetting of galinstan is good. The resistivity of the overall device is less than $0.1 \mathrm{ohm}$ and mainly due to wiring and connectors. However, we hope that some residual contact resistivity helps to bring a more homogeneous current along the axis. The symmetry and the homogeneity of this design have been tested by numerical computation. ${ }^{19}$ The top and bottom covers are made of polycarbonate implying an insulating boundary condition where the Hartmann layer is formed.

The height of the annular channel is $H=120 \mathrm{~mm}$. Galinstan is confined in a gap of $12 \mathrm{~mm}$ between the two cylindrical electrodes of radius $R_{1}=28 \mathrm{~mm}$ and $R_{2}=40 \mathrm{~mm}$. Thus, $\eta=0.7$ and the aspect ratio is $\Gamma=0.1$ (see Figure 1) as in some previous Taylor-Couette devices. ${ }^{20} \mathrm{We}$ especially checked that the radial inhomogeneity of the applied field is less than $0.5 \%$ within the fluid gap. Its axial variations are less than $4.5 \%$ and we take care to keep it symmetrical with respect to the mid-plane of the cell and to the axial alignment of all the apparatus. The coil, powered by a Sorensen DP 8-800 power supply and water-cooled, can reach a magnetic field up to $0.15 \mathrm{~T}$. The radial current crossing the cell is supplied by a Kepco BOP 20-50MG power supply providing up to $50 \mathrm{~A}$.

\section{B. Dimensionless parameters}

With a length scale equal to the fluid gap, $\delta=R_{2}-R_{1}$, a natural velocity scale appears from the dimensionless Navier-Stokes equation driven by the Lorentz force

$$
U_{o}=\sqrt{\frac{B_{o} I_{o} \delta}{R_{1} H \rho}}
$$

coming from a balance between the advection term and the Lorentz force effective at large Reynolds number. Notice that the balance of Lorentz force with the diffusion term, effective at low Reynold number, would give the linear scaling

$$
U_{o}^{\prime}=\frac{B_{o} I_{o} \delta^{2}}{R_{1} H \rho v} .
$$

This is actually the typical velocity scale appearing in Eq. (1). The order of magnitude of this velocity, for the highest forcing available, is: $U_{o} \approx 6 \mathrm{~cm} / \mathrm{s}$ and $U_{o}^{\prime} \approx 11 \mathrm{~m} / \mathrm{s}$ and the corresponding Reynolds number, $R e=U_{o} \delta / v \approx 2000$ and $R e^{\prime}=U_{o}^{\prime} \delta / v \approx 4 \times 10^{5}$. Obviously, the second scaling is inconsistent. Notice that a Reynold number constructed on the largest scale $H$ would have reached a value up to 20000 . The magnetic Reynolds number, $R m=\mu_{o} \sigma U_{o} \delta$, measuring the ratio of the characteristic time of magnetic diffusion, $\mu_{o} \sigma \delta^{2}$, over advection time, $\delta / U_{o}$, is less than $10^{-3}$ in our case. This shows that the magnetic field lines are almost unaffected by the flow. The interaction parameter, $N$, usually introduced to estimate the ratio of the induced Lorentz force $\sigma\left(u \times B_{o}\right)$ $\times B_{o}$ to the advection, $N=\sigma B_{o}^{2} \delta /\left(\rho U_{o}\right)$ is of order one in our case. The last relevant dimensionless parameter in our experiment is the Hartmann number, $H a=B_{o} H \sqrt{\sigma / \rho v}$ giving the size of the boundary layers of the velocity on the walls perpendicular to the magnetic field, $e_{H}=H / H a$. This is also the layer where all electrical currents are concentrated. Typically in our experiment, one get a $H a$ up to 460 and a Hartmann layer $e_{H}$ smaller than $0.3 \mathrm{~mm}$, i.e., $e_{H} / H \sim 2 \times 10^{-3}$. By the channelling of the currents near the top and bottom plate into the Hartmann layer, the magnetic field also generates a boundary layer near the vertical electrod, called the Shercliff layer, which is assumed to scale ${ }^{21}$ like $e_{S}=\delta / \mathrm{Ha}^{1 / 2}$. This layer is of order of $0.5 \mathrm{~mm}$.

One can assume that instabilities will occur when these boundary layers destabilize i.e., when the Reynold numbers built on their width, $R e_{H}=U_{o} e_{H} / v$ and $R e_{S}=U_{o} e_{S} / \nu$, overcome a given onset: $R e_{H}^{c} \lesssim 25$ and $R e_{S}^{c} \lesssim 65$, both based on energetical stability criteria. ${ }^{22}$ Therefore, we could expect 
instability across the lines $H a=\frac{R e}{\Gamma R e_{H}^{c}}$ and $H a=\frac{R e^{2}}{\left(R e_{S}^{c}\right)^{2}}$ in the plane $(H a, R e)$. Notice that in both cases the magnetic field stabilizes the flow. However, once the fluid moves under the action of the Lorentz force, one may consider consequences of the rotation on the flow. This can be estimated with the Elsasser number, balancing the Lorentz and centrifugal forces: $\Lambda=\frac{\sigma B_{o}^{2} \bar{R}}{2 \rho U_{o}}=\frac{H a^{2}}{R e} \Gamma \frac{\bar{R}}{H}$, where $\bar{R}$ is a characteristic radius of curvature of the cell, we took $\bar{R}=\left(R_{1}+R_{2}\right) / 2$. Therefore, one can expect an hydromagnetic driven instability for large $\Lambda$ and centrifugal driven instability at low $\Lambda$.

\section{Measurement device}

The physical properties of liquid metal, opacity, high thermal conductivity, and the high current density inside the cell, prevent the use of usual anemometry methods, such as hot wire, particles image velocimetry, or Laser Doppler velocimetry to study the flow. The previous studies used local or global potential measurements. ${ }^{10,13}$ However, to deduce the velocity field from this kind of measurement some assumptions are needed. ${ }^{14}$ We use here an ultrasonic Doppler velocimeter DOP3010 from Signal processing with two ultrasound probes to extract directly velocity profiles as function of the vertical coordinate. The measure is based on two Ultra-sound pulses (USP) emitted by the probe. The working frequency is around $4 \mathrm{MHz}$. The temporal resolution is limited by the time delay between successive pulses which depends on the expected velocity of the flow and the sound speed in the material. In our case it cannot be smaller than $0.1 \mathrm{~s}$. Nine locations have been arranged for our two probes on the top and bottom of the cell. The adjustment of the polycarbonate thickness with the ultrasonic wavelength, lets transmit an echo signal strong enough to get confident velocity estimations. Special care has been taken during the filling to prevent air bubbles. However, as the wetting of galinstan on the polycarbonate plate is not good, multi-reflections on this interface galinstan-polycarbonate, could polluted the first $10 \mathrm{~mm}$ of the measure. Each location has different angles, ranging from $0^{\circ}$ to $8^{\circ}$, with respect to the cylinder axis in order to be able to deduce the azimuthal and axial velocities. The USP are scattered by galinstan oxides present in the media. Ultrasonics probes give the projection on their axis of the velocity profile. This profile is estimated by a sampling of 550 measurement gates at different location along the probe axis, corresponding to a spatial resolution of $1 / 4 \mathrm{~mm}$. It is worth noticing that the volume explored by the probe is not homogeneous along the axis. There is a tightening of the beams up to the near field limit around $d^{2} f(4 c) \sim 2.5 \mathrm{~cm}$ with $d$ the probe diameter, $f$ the probe frequency, and $\mathrm{c}$ the sound speed in galinstan. Then the beam width diverges with an angle $\alpha \sim c /(f d)=4.5^{\circ}$ due to diffraction.

\section{Experimental procedure}

Benchmarks of ultrasonic velocity measurement in gallium and gallium alloys with respect to other fluids and other measurement probes, underline possible problems with gallium oxides. ${ }^{15,17}$ Although they are used as scatters, they can accumulate near the sensor and decrease the signal quality. In order to have well-reproducible results with the USP, it is necessary to get homogenized scatters in galinstan. As the main oxide $\mathrm{Ga}_{2} \mathrm{O}_{3}$ seems to have an almost equal or slightly higher density than the fluid, ${ }^{23}$ we drive the fluid to the highest speed available (with $I_{o}=50 \mathrm{~A}$ and $B_{o}$ $=1000 \mathrm{G}$ ) during $20-60 \mathrm{~s}$ them before each measurement. That insures the mixing of the oxides. Then we let the flow relax during 30-60 s at the value $I_{o}, B_{o}$, prescribed for the measurement before starting the measurement. This procedure and our filling procedure, where we try to remove all the contact with air, in addition to the fact that most of the presented measurements are made from the top plate, prevent the accumulation of large among of oxides near the sensor.

\section{RESULTS}

\section{A. Global behaviours of the flow}

Figure 2 shows the velocity profiles, averaged in time, of probes with angles of $8^{\circ}-V_{1}$ (left) - and $0^{\circ}-V_{2}$ (right) - these probes are located at a radius $R_{\text {probes }}=\left(R_{1}+R_{2}\right) / 2$ and are separated 

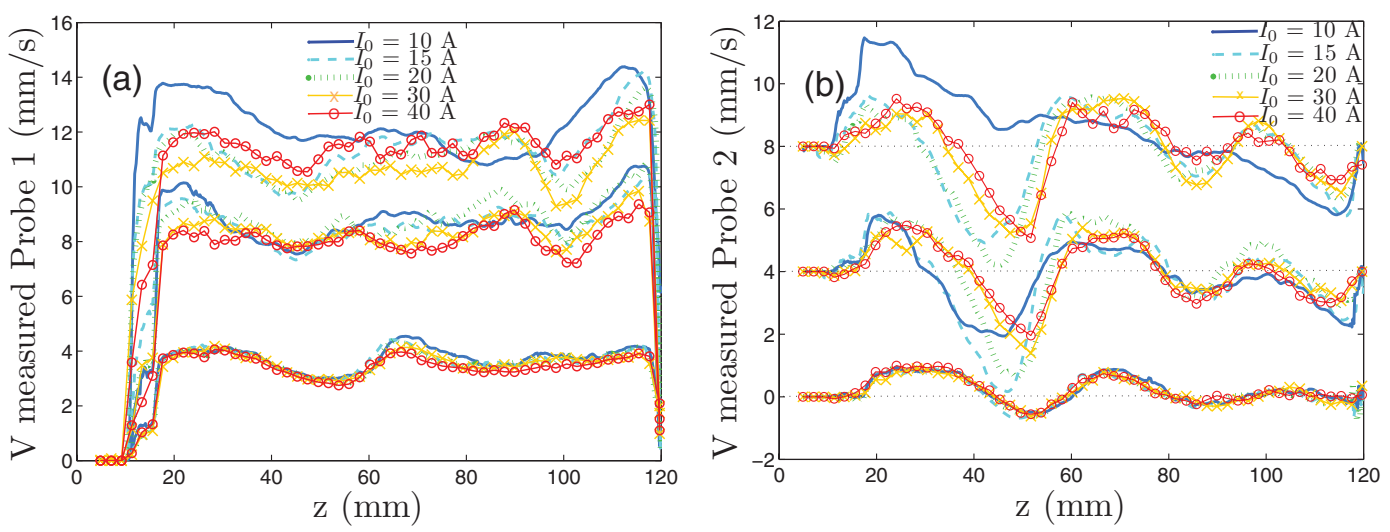

FIG. 2. Time averaged velocity profiles (over 200 snapshot) - for $I_{o} \cdot B_{o}=1000,3000,5000$ G.A (corresponding to $R e$ $=[\sim 670, \sim 1670$, and $\sim 2670]$ ) at different cell current (Icell $=10,15,20,30$, and $40 \mathrm{~A}$ ) of the probes at $8^{\circ}$ (a) and at $0^{\circ}(\mathrm{b})$. Note that on the right, curves have been shifted for clarity, the dash line gives the value of the shift from 0 . The value of the shift increases when the magnetic field increases.

by an angle of $2 \pi / 3$. Each curve is the average of 200 instantaneous profiles. Three values of the forcing product $I_{o} \cdot B_{o}=1000,3000,5000$ G.A are plotted in Figure 2 (from the bottom to the top) and for each value $I_{o} \cdot B_{o}=$ constant, 4 values of the current are represented. As expected, the profiles, $V_{1}$ of the probe with an angle, increase with the forcing since they measure a part of the tangential velocity $\overline{u_{\theta}}$. Due to the Hartmann layer each tangential profile is nearly flat in the bulk. It can be noticed that for low forcing, profiles seem independent of the individual values of $I_{o}$ and $B_{o}$ as long as their product is constant. This is not true anymore at high forcing. It is worth noticing that the velocity near the upper wall in the near field limit, i.e., for $z<20 \mathrm{~mm}$, is polluted by the multi-reflection of the sound wave at the plexiglas galinstan interface.

The profiles of the axial velocity component are almost 0 in average, but we represent them shifted in Figure 2 in order to clarify the effect of the magnetic field. One can notice that the fluctuations of underlining structures in the axial velocity field do not vanish after time averaging. Structures of the same order are actually also present on the other probe. The wavelength of these structures seems to evolve with the magnetic field. Such a structure can be explained by a secondary flow. It can be a consequence of a first stationary instability but we do not succeed to catch the threshold of this first instability. Indeed, measurements at small velocity are difficult mainly because long measurements are necessary and it is more difficult to prevent the sedimentation of the scatters. It could be also the consequence of tangential and axial inhomogeneity of the current induced by the Hartmann boundary layer. Indeed as soon as $e_{H}$ is smaller than $H$ then the current is not anymore only radial, some lateral boundary Shercliff layers develop, scaling with $1 / \sqrt{B} \cdot{ }^{21}$ Then the forcing can be not only tangential.

In spite of the care brought to keep the experimental device symmetrical, velocity profiles are not symmetrical between the top and bottom parts of the cell. This can be due to some measurement problems, indeed the ultrasonic beam is not symmetrical, the scatter density can be inhomogeneous, etc. It can also come from flow asymmetry or from both effects. For instance, gradient density of the scatters can change the velocity detection but can also modify the fluid properties. In order to pick out between these hypotheses, a probe has been placed under the bottom plexiglas plate. In this case, the structure appears stronger near the bottom, i.e., near the sensor. This tends to prove that the asymmetry is mainly due to measurement problems although the symmetry is not entirely recovered in this case. For practical reasons, most of the measurements have been performed with sensors at the top where they are most easy to adjust. Therefore hereafter, we will focus our attention on the phenomena occurring on the first half of the cell.

With two probes, we are able to rebuild the mean tangential and axial velocity. To prevent boundary effects, the average is estimated only in the bulk between $z=30 \mathrm{~mm}$ and $z=90 \mathrm{~mm}$. 


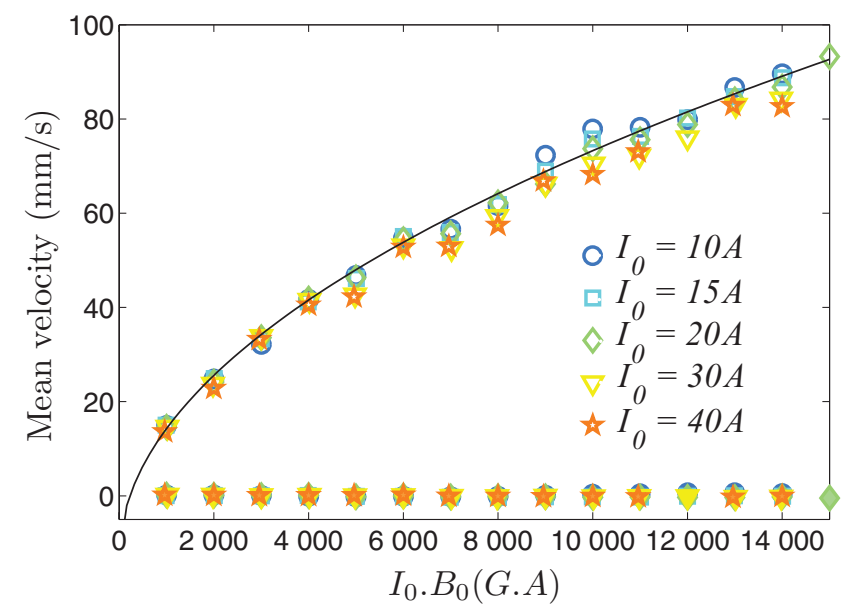

FIG. 3. The mean velocity along the tangential axis, $\left\langle\overline{u_{\theta}}\right\rangle$ (open symbols) and near 0 , along the $\mathrm{z}$ axis, $\left\langle\overline{u_{z}}\right\rangle$ (full symbols), as a function of the forcing $F_{o}=B_{o}, I_{o}$. Spatial mean is estimated between $z=30 \mathrm{~mm}$ and $z=90 \mathrm{~mm}$. The colors represent the different current intensity for a given $F_{o}$. The full line is the best square root fit of the azimuthal velocity.

These mean values for both components are plotted in Figure 3 as a function of the forcing $F_{o}=B_{o} \cdot I_{o}$. Colored symbols deal with the imposed intensity for a given $F_{o}$. The tangential component increases with the forcing, whereas the axial does not. The magnitude of $\left\langle\overline{u_{\theta}}\right\rangle$ is of order of the crude one, obtained by dimensional analysis in Sec. II B and the growth is compatible with an expected square root behaviours $\left\langle\bar{u}_{\theta}\right\rangle \sim a \sqrt{I_{o} \cdot B_{o}}-b$ with $a=0.86$ and $b=13$ (b is necessary because near $F_{o} \sim 0$ a viscous linear behaviour is expected). Such a scaling, corresponding to a balance between advection and Lorentz force, was expected in our range of Reynolds number (from 600 to 3000). However, as noticed on the previous figure, points are more scattered when the forcing is increased. It seems that the mean tangential velocities tend to increase with magnetic field at a given $F_{o}$. For instance at $F_{o}=10000$ A.G the mean tangential velocity is about $8 \mathrm{~mm} / \mathrm{s}$ (i.e., $10 \%$ ) smaller for $I_{o}=40 \mathrm{~A}$ and $B_{o}=250 \mathrm{G}$ (orange stars) than for $I_{o}=10 \mathrm{~A}$ and $B_{o}=1000 \mathrm{G}$ (dark blue circles). The mean axial velocity is hopefully nearly 0 .

We complete these results by the evaluation of the fluctuations of the profiles. The root mean square (rms) value of the time average velocity profiles, $\sigma\left(\overline{u_{\theta}}\right)$, in the gap $z=30 \mathrm{~mm}$ to $z=90 \mathrm{~mm}$ is computed. Results are shown in Figure 4 as a function of the forcing $F_{o}$ for various intensities. For low forcing, the fluctuations grow with the forcing. However, for the smaller current of Figure 4, i.e., $I_{o}=10 \mathrm{~A}$ (blue dark circles), fluctuations reach a maximum value for $F_{o}$ $=6000$ A.G. This is also observed for $I_{o}=15 \mathrm{~A}$ (cyan squares) where the maximum is reached at higher forcing, i.e., for $F_{o}=8000$ A.G. This can be understood as a stabilizing effect of the magnetic field (which can be large in these cases) although it increases the driving Lorentz force. Fluctuations continuously grow when $I_{o}$ is large. Then, at large forcing, the magnetic field stays moderated and cannot compensate the fluctuations induced by the growth of the forcing. In this case, relative fluctuations are almost constant with $\sigma\left(\overline{u_{\theta}}\right) /\left\langle\overline{u_{\theta}}\right\rangle \sim 0.02-0.025$. In contrast to the mentioned observation for the mean value in Figure 3, for a given large $F_{o}$, fluctuations are now around $2 \mathrm{~mm} / \mathrm{s}$ larger for $I_{o}=40 \mathrm{~A}$ and $B_{o}=250 \mathrm{G}$ (orange stars) than for $I_{o}=10 \mathrm{~A}$ and $B_{o}=1000 \mathrm{G}$ (dark blue circles). By this comparison of Figures 3 and 4, it seems that a part of the kinetic energy of the fluctuations is transferred to the mean flow when the magnetic field is increased at a given $F_{o}$. The forcing is then more efficient to improve the mean flow rate.

It has to be noted that the drop in the rms value for $I_{o}=10,15$ (which is also present for $I_{o}$ $=8,12$, but not represented here) corresponds to a change in the $V_{z}$ mean profile measure. Indeed, as shown in the top curves of Figure 2 (right), the mean profile presents 3 maxima initially (curves from orange to cyan), but only one subsists after the rms drop (curve dark blue). 


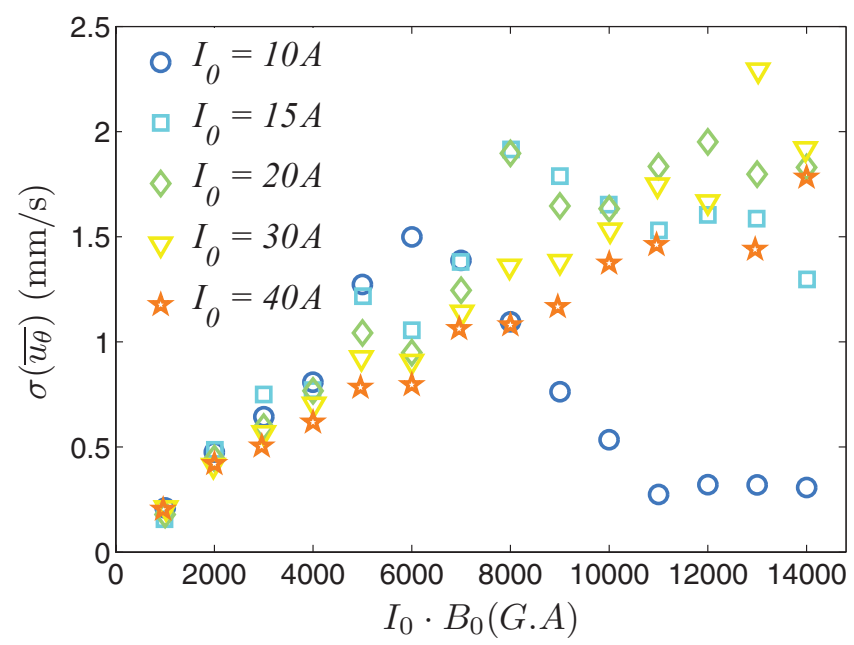

FIG. 4. The standard deviation of the spatial fluctuation of the time averaged velocity profiles along the tengential axis, $\sigma\left(\overline{u_{\theta}}\right)$, as a function of the forcing $F_{o}=B_{o} \cdot I_{o}$. The root mean square $(\mathrm{rms})$ is estimated between $z=30 \mathrm{~mm}$ and $z$ $=90 \mathrm{~mm}$. The color represents the different current intensity for a given $F_{o}$.

\section{B. Dynamical regimes}

In order to have better understanding of this process of restabilization, we studied the time evolution of instantaneous velocity profiles.

Figure 5 shows the spatiotemporal diagrams of the axial velocity for $I_{o}=10 \mathrm{~A}$ and $B_{o}=200$, 700,800 , and $1200 \mathrm{G}$. In spite of the background noise, Figure 5(a), for which $B_{o}=200 \mathrm{G}$, exhibits only a stationary spatial structure around $z=55 \mathrm{~mm}$ (and a slighter around $z=85 \mathrm{~mm}$ ). By increasing the magnetic field further, it appears that this structure becomes unstable and generates some not perfectly periodic propagative waves as shown in Figure 5(b) for $B_{o}=700 \mathrm{G}$. The wavelength seems to decrease when the magnetic field is increased as illustrated in Figure 5(c) for $B_{o}=800$ G. Finally for higher field, spatial structures almost disappear whereas waves of higher frequency emerge on Figure 5(d), where $B_{o}=1200 \mathrm{G}$. Following these observations, one can expect that the maximum observed on the fluctuations shown in Figure 4 for $I_{o}=10 \mathrm{~A}$ would be smoothed out by longer time averaging. However, it could keep trace of the stationary structure observed a lower magnetic field which is destabilized by these waves.

In order to confirm these observations, we have plotted the power spectral density, PSD, of the temporal trace at $I_{o}=10 \mathrm{~A}$ in Figure 6 for different values of the magnetic field. These PSDs have been averaged in space over gates between $z=20$ and $z=70 \mathrm{~mm}$. It underlines the frequency change of waves observed in Figure 5. There are no significant frequencies at low driving (blue curves) i.e., for $B_{o} \leq 600 \mathrm{G}$. A peak at a low frequency, $f_{1}$, appears near $f_{1}=0 \mathrm{~Hz}$ (less than $15 \mathrm{mHz}$ in our case) for $B_{o}$ slightly below $600 \mathrm{G}$ corresponding to the first travelling waves, TW1, observed in Figures 5(b) and 5(c). The amplitude of this frequency first grows and then decreases with increasing $B_{o}$. This frequency also slightly shifts to higher values (up to $45 \mathrm{mHz}$ ). When the amplitude of this frequency starts to decrease another higher peak starts to grow around finite frequency $f_{2} \sim 195 \mathrm{mHz}$. This appears more clearly in Figure 6 where the excess of the amplitude $A^{*}$ of the low frequency (i.e., $<0.1 \mathrm{~Hz})$ and high frequency $(>0.1 \mathrm{~Hz})$ peaks are plotted in blue and red, respectively. Clearly, the low frequency peak starts to grow around $B_{o}=600 \mathrm{G}$ and reaches a maximum around $800 \mathrm{G}$. At that magnetic field, the high frequency peak starts to grow. Above $1200 \mathrm{G}$ only this last peak remains. For these waves, the frequencies evolve slightly from $195 \mathrm{mHz}$ to $222 \mathrm{mHz}$ when the magnetic field is increased from $800 \mathrm{G}$ to $1200 \mathrm{G}$.

Then we consider the time correlation at different locations in space along the $30<z<70 \mathrm{~mm}$ axes which underlines the different nature of the observed waves. In Figure 7 is plotted the time 

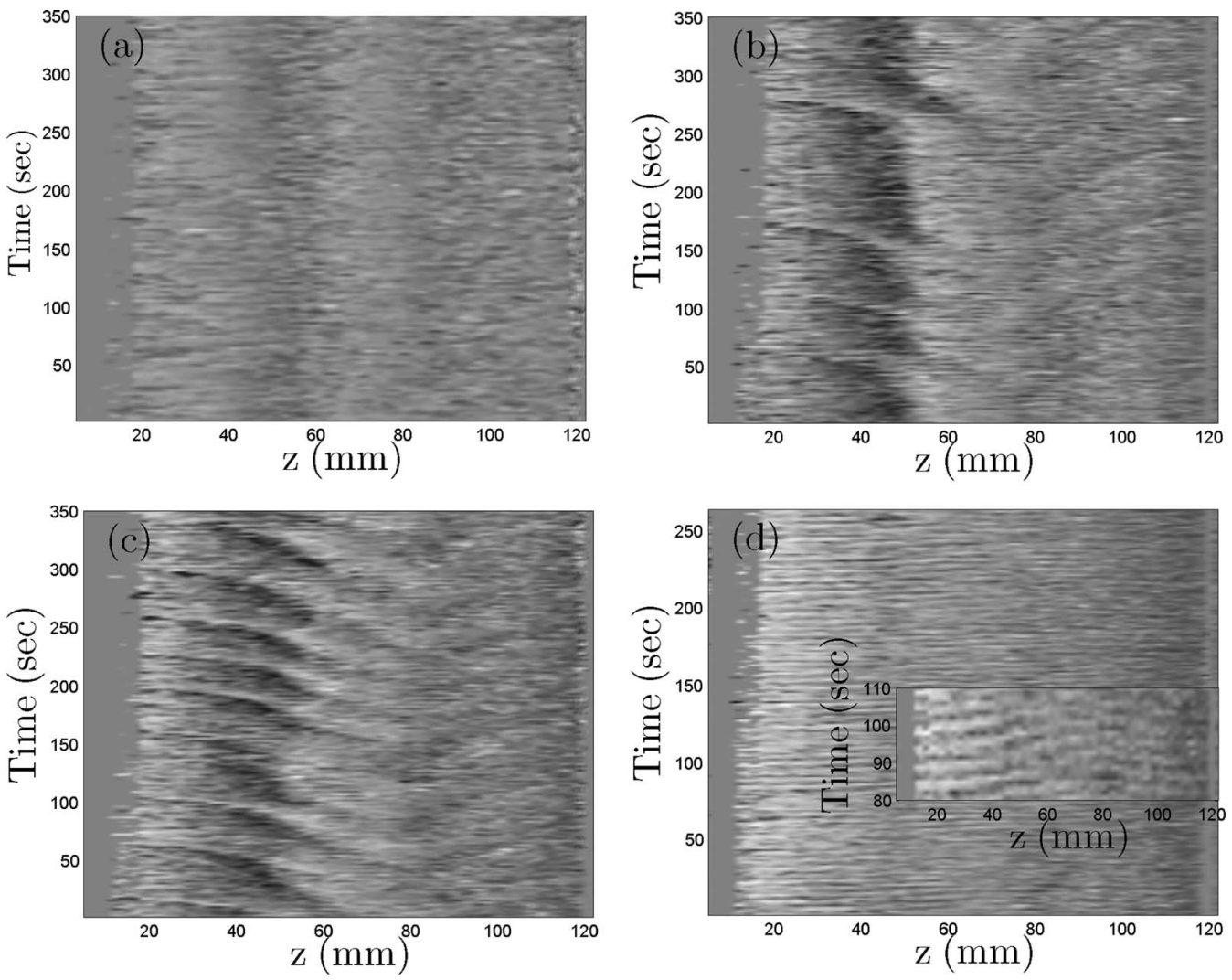

FIG. 5. Spatiotemporal diagrams of the axial velocity, $u_{z}$, for different forcing regimes for $I_{o}=10 \mathrm{~A}$ and (a) $B_{o}=200 \mathrm{G}$, (b) $B_{o}=700 \mathrm{G}$, (c) $B_{o}=800 \mathrm{G}$, (d) $B_{o}=1200 \mathrm{G}$ (the colormap is between -8 to $8 \mathrm{mms}^{-1}$ ). On this last figure a zoomed view is shown in the inset.

correlation for different distance between measurement gate in a 2D plot. Each distance interval is average on all the gates. A low pass filter of $2 \mathrm{~Hz}$ is applied to the signal. The travelling waves appear clearly in this representation. It is worth noticing that the low frequency wave (left), TW1, and high frequency (rigth), TW2, are actually both propagative but in different directions. In the upper half part of the cell we consider, waves always keep the same direction. However, it seems that they both propogate in the other direction of the lower part, although we do not perform systematic study with probes at the bottom. Notice that, at our level of accuracy, the correlation measurements between two ultrasonic probes at different azimuthal angles do not reveal other correlation time than the one related to advection at the mean azimuthal velocity.

To confront these waves with others expected in MHD, it is useful to estimate the Lundquist number $L=\sigma \sqrt{\mu_{o} / \rho} B_{o} \delta \sim 7 \times 10^{-2}$ at the highest magnetic field. Indeed, only for large enough $L$ can Alfven wave propagate, because $L$ is also the magnetic Reynolds number based on the velocity of Alfven waves $V_{a}=B_{o} / \sqrt{\mu_{o} \rho} \sim 1.3 \mathrm{~m} / \mathrm{s}$. Such fast waves would be difficult to measure in our device anyway. From the slope of the spatiotemporal structure we can deduce a velocity of propagation as shown in Figure 8 as a function $B_{o}$ for TW $1 I_{o}=10$. This speed, $c_{1}$, increases with $B_{o}$ but never overcomes the $\mathrm{mm} / \mathrm{s}$. For fixed magnetic field, the velocity increases with the current. Typically for $B_{o}=600 \mathrm{G}$, the speed grows linearly form $c_{1}=0.26 \mathrm{~mm} / \mathrm{s}$ at $I_{o}=10$ A to $c_{1}$ $=0.815 \mathrm{~mm} / \mathrm{s}$ at $I_{o}=20 \mathrm{~A}$. In the regime TW2, a speed, $c_{2}$ of order of the $\mathrm{cm} / \mathrm{s}$ is observed. However, in our range of parameters available in this regime and with our level of accuracy there is no clear dependency of $c_{2}$ with the forcing. The low frequencies (less than $1 \mathrm{~Hz}$ ) observed in our device are of the same order as some frequencies observed in secondary instability like the wavy vortex of the Taylor-Couette flow. ${ }^{24}$ However, it is difficult to do quantitative comparison because the forcing and 

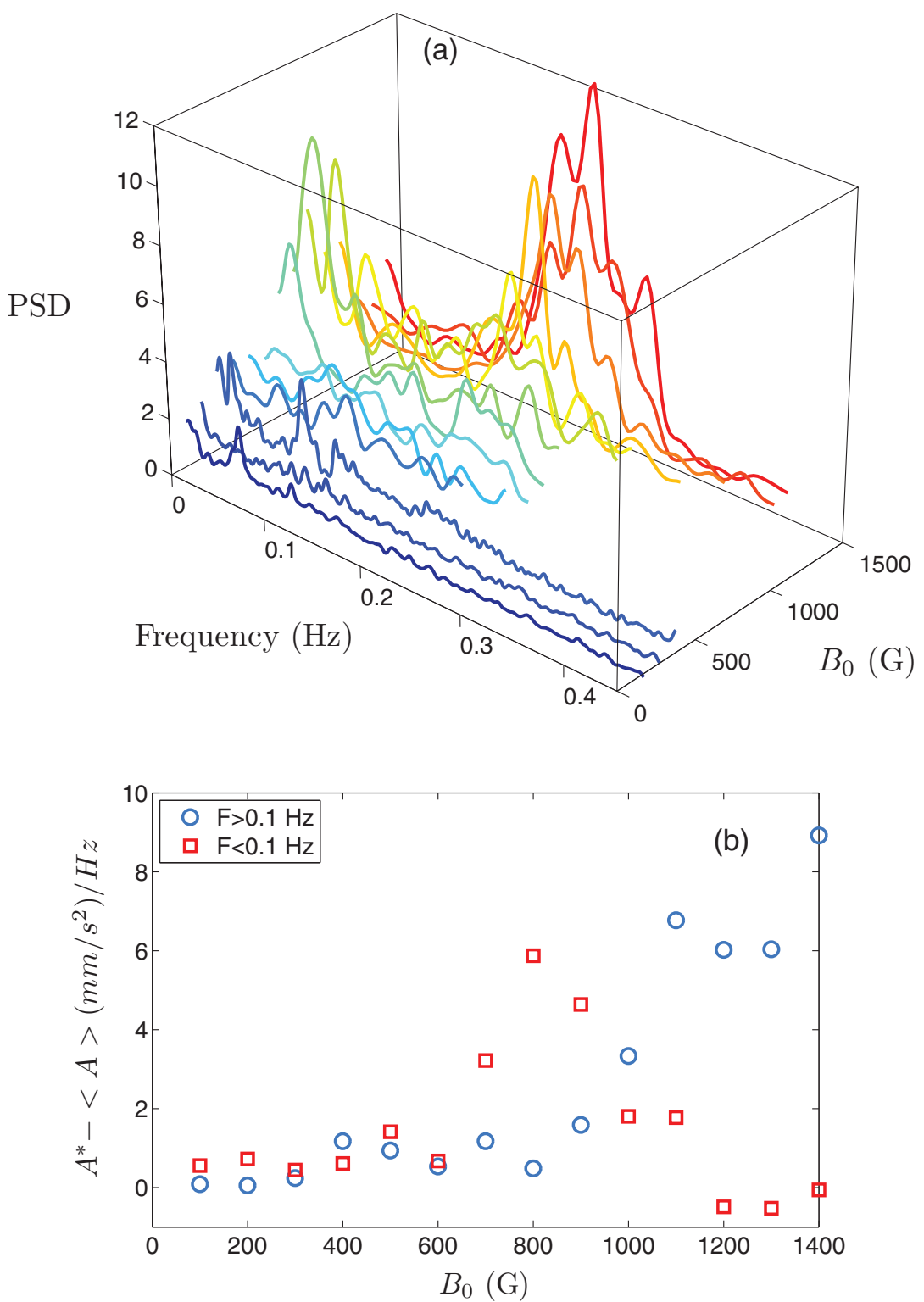

FIG. 6. (a) Temporal spectrum of the vertical probe, spatially averaged between $20<z<50$ for $I_{o}=10 \mathrm{~A}$ and magnetic field $B_{o} \in[200$ 1200] $\mathrm{G}$ from the blue to the red curve. (b) Evolution of the amplitude of highest peak of the PSD for frequencies smaller than $0.1 \mathrm{~Hz}$ (red squares) and larger than $0.1 \mathrm{~Hz}$ (blue circles).

geometry are different. It is worth noticing that for both waves, the wavelength defined as $\lambda_{i}=c_{i} / f_{i}$, $i=1,2$, is of order of the $\mathrm{cm}$, i.e., of order of the fluid gap.

These transitions from stationary structure to TW1 and from TW1 to TW2, is also observed at $I_{o}$ $=8 \mathrm{~A}$ and $I_{o}=12 \mathrm{~A}$. For higher currents we observe only the low frequency travelling waves, TW1, in the range of magnetic field accessible in our experiment. At the highest forcing available no wave is observed. Therefore, we are able to establish the state diagram shown in Figure 9 where different symbols mark the presence of no frequency, low frequency $(<0.1 \mathrm{~Hz})$, and higher frequency peaks. Modulated waves occur at the overlaps. One can notice that when the magnetic field is fixed and the current is increased, then the fast travelling waves (TW2) can be modulated by the slower, as clearly shown in the horizontal line around $H a=182$. We can suspect that restabilization occurs at higher magnetic field, but this deserves further studies. 
(a)

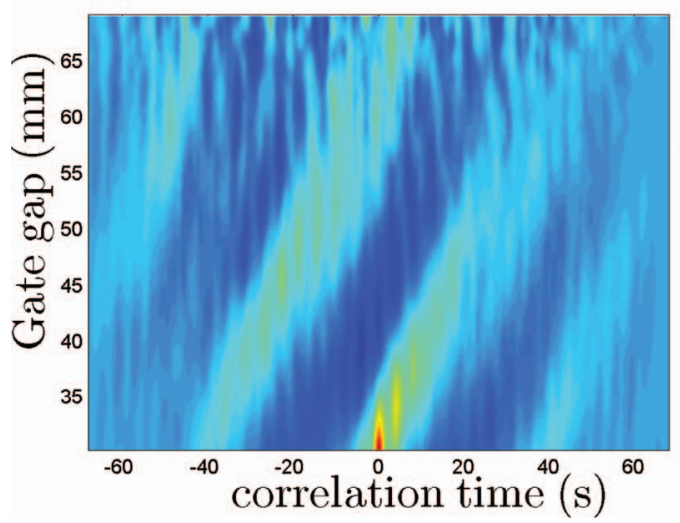

(b)

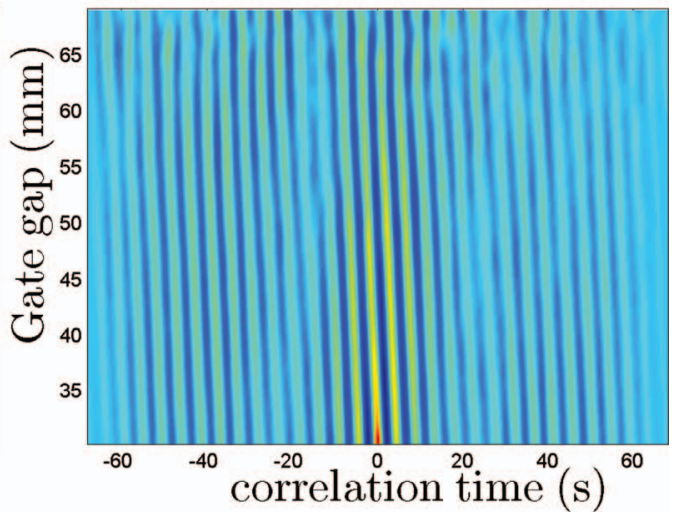

FIG. 7. 2D plot of the time correlation of waves at different location in space along the $z$ axis. (a) TW 1 for $I_{o}=10 \mathrm{~A}$ and $B_{o}=880 \mathrm{G}$ and (b) TW2 at $I_{o}=10 \mathrm{~A}$ and $B_{o}=1200 \mathrm{G}$.

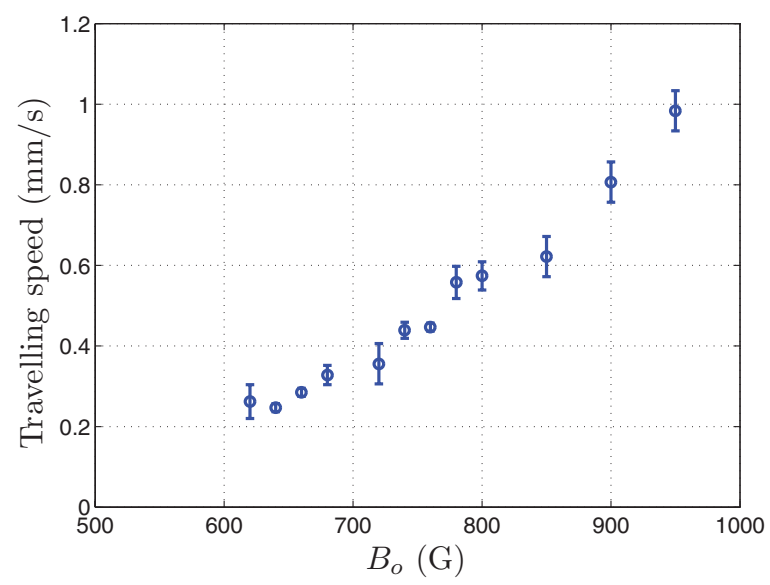

FIG. 8. Travelling velocity of the TW1 wave as a function of the applied magnetic field for $I_{o}=10 \mathrm{~A}$.

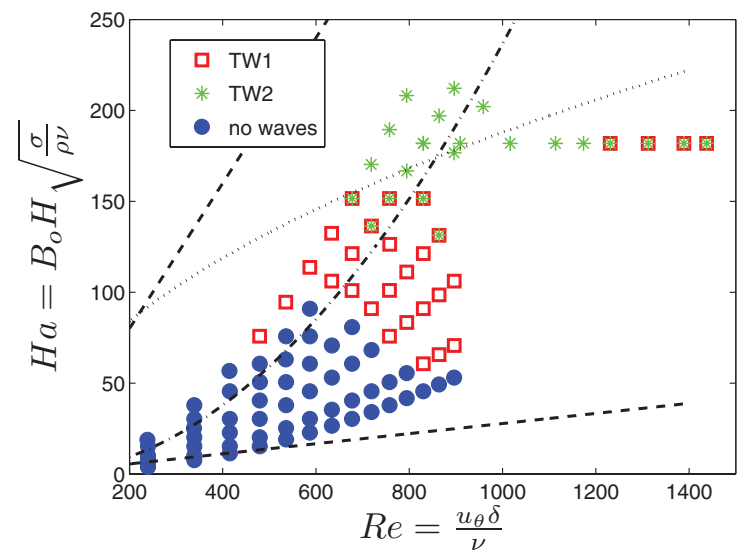

FIG. 9. Phase diagram of the explored dynamical regimes. Symbols: Blue disks no oscillation, open red squares low frequency oscillation peak (TW1), green stars high frequency oscillation peaks (TW2). The two straight dashed lines correspond to the curve $H a=R e /\left(\Gamma R e_{H}^{c}\right)$ over which Hartmann layer is stabilized, with $R e_{H}^{c}=25$ for the upper one and $R e_{H}^{c}=390$ for the lower. The dotted-dashed line corresponds to $H a=\left(R e / R e_{S}^{c}\right)^{2}$ over which Shercliff layer is stabilized, the dot line $H a=\sqrt{\operatorname{ReH} /(\Gamma \bar{R})}$ below which the rotation dominates. 


\section{DISCUSSION AND CONCLUSIONS}

We have performed an experiment where a conducting fluid is forced electromagnetically between two cylindrical coaxial electrodes providing a radial current and an axial magnetic field. Our aim was to underline the respective role of the parameters entering in the forcing: the current and magnetic fields. We found that

- the time averaged velocity profile is mainly along the tangential component and shows fluctuations smaller than $20 \%$ (based on the maximum and minimum of the fluctuation).

- The mean tangential velocity grows approximatively like the square root of the forcing $F_{o}=I_{o} \cdot B_{o}$ corresponding to a fully nonlinear regime.

- a closer study shows that for a given $F_{o}$, a growth of the magnetic field, induces a growth of the mean flow and reduces the fluctuations.

- We exhibit dynamical regimes starting from a stationary structure present at high currents and low magnetic fields.

- There is an intermediate regime where the structure seems destabilized and the system shows low frequency travelling waves (starting at nearly $0 \mathrm{mHz}$ and going up to $45 \mathrm{mHz}$ when the magnetic field is increased).

- Finally, when the magnetic field is high, these travelling waves decline and another kind of travelling waves grows at higher frequency (around $200 \mathrm{mHz}$ ).

It is important to distinguish these phenomena from various MHD instabilities existing in similar geometries. Because of its implication in astrophysics, the most studied instability is probably the Magneto-Rotational Instability (MRI). ${ }^{25,26}$ Starting with a purely stable tangential velocity field forced mechanically, it has been shown that a magnetic field perpendicular to the velocity field destabilizes the flow and enhances the momentum transport. Stable azimuthal velocity profile against the Rayleigh criterion $\partial\left(r \cdot u_{\theta}\right) / \partial r>0$ is a precondition to relevancy of the MRI. We are in a different case in our system. Indeed as long as the induction is neglected, the velocity profile in Eq. (1) does not satisfy the Rayleigh stability criterion; due to the motionless outer wall it should be also the case when induction is considered. ${ }^{9}$ However, it is worth noticing that the velocity of the waves TW1 is of order of the one predicted and observed in Helical MRI. ${ }^{27}$ The main difference is that in our case, the wave does not grow from purely tangential flow since a stationary structure is already there. Other magneto-inertial waves have been detected in the Derviche Tourneur Sodium (DTS) experiment. ${ }^{28}$ Although there are no applied current nor tunable magnetic fields in this spherical-Couette flow, the shear and the global rotation imposed to the fluid can be controlled independently. The reduction of the number of tangential modes when global rotation is included could have some connection with the reduction of fluctuations observed when the magnetic field is increased. Indeed rotation and magnetic fields both imply a bi-dimensionalization of the flow.

In order to distinguish rotational and magnetic effects on the instabilities of the flow, we also plot on Figure 9, the lines corresponding to the laminarisation of the Hartmann layer (dashed line), the laminarisation of the Shercliff layer (dotted-dashed line), and the dotted line $\Lambda=1$ below which the centrifugal effects dominate. The precise value of the critical value $\operatorname{Re}_{S}^{c}$ and $R e_{H}^{c}$ is still debated $^{22}$ and should depend on the precise geometry of the device. For instance in a straight pipe, ${ }^{22}$ an energetic criterion guarantees stability of the Hartmann layer for $R e_{H}^{c}<25$, whereas linear analysis predicts $R e_{H}^{c}=48000$, and numerical simulation with nonmodal pertubations observed instabilities for $R e_{H}^{c} \geq 390$. In Figure 9, we choose $R e_{S}^{c}=65$ and $R e_{H}^{c}=25$ or $R e_{H}^{c}=390$. Despite these uncertainties, it is tempting to assume that instability of the Hartmann layer is irrelevant for our observations. The TW1 waves could be controlled only by hydro-rotational effect since they occur essentially for $\Lambda<1$. The waves TW2 may imply magnetic effects, since they appear around the boundary where the Shercliff layer is stabilized, and rotational effects. Indeed, it is known that even for $\Lambda>1$, the rotational effects can play a role in determining stability of the flow at least for the Bödwadt-Hartmann boundary layer problem. ${ }^{29}$ Obviously, this assumption needs a lot of further theoretical and experimental works. For instance, the role of the cell geometry, aspect ratio, and curvature, on the stability of the boundary layers deserves more study which could be helpful to discern the different aspects of the observed instability. 
It would also be interesting to connect these mechanisms to instabilities explored in Ref. 13 by the measurement of global potential applied through the cell. We plan to perform such global measurements to try to catch traces of the instabilities in the injected power. The torque applied by the moving fluid to the confining walls is another interesting global quantity to connect to the observed instabilities. Such a measurement performed in Taylor-Couette flow reveals puzzling results. ${ }^{31,32}$ In our case, this measurement could be facilitated by the fact that the walls are motionless.

Concerning purely electromagnetically forced flows, one can first notice that a low frequency instability has been reported in large electromagnetic pump. ${ }^{30}$ Although the geometry based only on induction by a time-dependent magnetic field is quite different, one can think that the analysis of our simplified design can help to characterize the role of current and magnetic fields in such instabilities. It is difficult to match our experiment with the one presented in Ref. 11, because of their differences: larger aspect ratio, strongest magnetic field, different working fluids. However, it is the closest from our device. Beyond the different range of explored forcing parameters, it is worth noticing that similar behaviours still exist. Our direct measurements of the flow velocity along the axis reveal the structure underlying the flow and should be helpful to understand the instability mechanism. However, it will be useful to implement some local potential probes in our cell to facilitate the comparison and increase the time resolution. Finally, it is tempting to try to describe the observed instabilities in the framework of weakly nonlinear phenomena and amplitude equations with few modes in interaction that are able to describe various dynamical regime as shown even recently in Ref. 33. During the reviewing processes, we noticed a publication on numerical simulations of a similar device that deserved a close comparison with our experimental results. ${ }^{34}$

\section{ACKNOWLEDGMENTS}

We would like to thank C. Gasquet for helping the automation of the experiment, C. Normand for discussions and bibliographic advices, and M. Bonetti, O. Dauchot, B. Dubrulle, and Pablo Gutierrez-Matus for helpful discussions. We are indebted to Dr. J. C. Willemetz, Chairman of Signal Processing SA, for his advices on ultrasonic measurements. J.B. receives financial support from the Grant No. ANR05-0268-03.

${ }^{1}$ H. K. Moffatt, Magnetic Field Generation Electrically Conducting Fluids (Cambridge University Press, Cambridge, 1978).

${ }^{2}$ P. A. Davidson, "Magnetohydrodynamics in materials processing," Annu. Rev. Fluid Mech. 31, 273 (1999).

${ }^{3}$ J. A. Shercliff, A Textbook of Magnetohydrodynamics (Pergamon, Oxford, 1965).

${ }^{4}$ S. Chandrasekhar, Hydrodynamic and Hydromagnetic Stability (Dover, New York, 1961).

${ }^{5}$ J. Sommeria and R. Moreau, "Why, how, and when, MHD turbulence becomes two-dimensional," J. Fluid Mech. 118, 507 (1982).

${ }^{6}$ T. S. Chang and W. K. Sartory, "On the onset of instability by oscillatory modes in hydromagnetic Couette flow," Proc. Roy. Soc. A 301, 451 (1967)

${ }^{7}$ D. R. Sisan, N. Mujica, W. A. Tillotson, Y. M. Huang, W. Dorland, A. B. Hassam, T. M. Antonsen, and D. P. Lathrop, "Experimental observation and characterization of the magnetorotational instability," Phy. Rev. Lett. 93, 114501 (2004).

${ }^{8}$ F. Stephani, T. Gundrum, G. Gerbeth, G. Rüdiger, M. Schultz, J. Szklarski, and R. Hollerbach, "Experimental evidence for magnetorotational instability in a Taylor-Couette flow under the influence of a helical magnetic field," Phy. Rev. Lett. 97, 184502 (2006)

${ }^{9}$ R. M. Digilov, "Making a fluid rotate: Circular flow of a weakly conducting fluid induced by a Lorentz body force," Am. J. Phys 75, 361 (2007).

${ }^{10} \mathrm{P}$. Tabeling and J. P. Chabrerie, "Magnetohydrodynamic Taylor vortex flow under a trans-verse pressure gradient," Phys. Fluids 24, 406 (1981).

${ }^{11}$ P. Tabeling, "Sequence of instabilities in electromagnetically driven flows between conducting cylinders," Phys. Rev. Lett. 49, 460 (1982).

12 J. A. Baylis and J. C. R. Hunt, "MHD flow in an annular channel: Theory and experiment," J. Fluid Mech. 48, 423 (1971).

${ }^{13}$ P. Moresco and T. Alboussière, "Experimental study of instability of the Hartmann layer," J. Fluid Mech. 504, 167 (2004).

${ }^{14}$ J. A. Shercliff, The Theory of Electromagnetic Flow Measurement (Cambridge University Press, Cambridge, 1987).

${ }^{15}$ D. Brito, H. C. Nataf, Ph. Cardin, J. Aubert, and J.-P. Masson, "Ultrasonic Doppler velocimetry in liquid gallium," Exp. Fluids 31, 653 (2001)

${ }^{16} \mathrm{~S}$. Eckert and G. Gerberth, "Velocity measurements in liquid sodium by means of ultrasound Doppler velocimetry," Exp. Fluids 32, 542 (2002)

${ }^{17}$ O. Andreev, Y. Kolesnikov, and A. Thess, "Application of the ultrasonic velicity profile method to mapping of liquid metal flows under the influence of non-uniform magnetic field,” Exp. Fluids 46, 77 (2009). 
${ }^{18}$ From The safety datasheet acc, Guideline 93/112/EC of Germatherm Medical AG, galinstan is made of $68.5 \%$ of Gallium, $21.5 \%$ of indium, $10 \%$ of Tin. Its density is $\rho=6.440 \times 10^{3} \mathrm{~kg} / \mathrm{m}^{3}$, its kinematic viscosity is $v=3.73 \times 10^{-7} \mathrm{~m}^{2} / \mathrm{s}$, its electrical conductivity $\sigma=3.46 \times 10^{6}(\mathrm{~S} / \mathrm{m})$.

${ }^{19}$ A. Klochko, Instabilité dans les écoulements de fluides conducteurs forcés électomagnétiquement., Master 1 internship research report, Ecole Polytechnique, 2010.

${ }^{20}$ D. P. Lathrop, J. Fineberg, and H. L. Swinney, "Transition to shear-driven turbulence in Couette-Taylor flow," Phys. Rev. A 46, 6390 (1992).

${ }^{21}$ J. Hunt and K. Stewartson, "Magnetohydrodynamic flow in a rectangular duct. II," Fluid Mech. 23, 563 (1965)

22 A. Potherat, "Quasi-two-dimensional pertubations in duct flows under transverse magnetic field," Phys. Fluids 19, 074104 (2007).

${ }^{23}$ N. B. Morley, J. Burris, L. C. Cadwallader, and M. D. Nornberg, "GaInSn usage in research laboratory," Rev. Sci. Instrum. 79, 056107 (2008).

${ }^{24}$ C. D. Andereck, S. S. Liu, and H. L. Swinney, "Flow regimes in a circular Couette system with independently rotating cylinders," J. Fluid Mech. 164, 155 (1986).

${ }^{25}$ S. Chandraskhar, "The stability of non-dissipative Couette flow in hydromagnetics," Natl. Proc. Acad. Sci. U.S.A. 46, 253 (1960).

${ }^{26}$ S. A. Balbus and J. F. Hawley, "Instability, turbulence, and enhanced transport in accretion disks," Rev. Mod. Phys. 70, 1 (1998).

${ }^{27}$ G. Rüdiger, R. Hollerbach, F. Stefani, T. Gundrum, G. Gerbeth, and R. Rosner, "The travelling-wave MRI in cylindrical Taylor-Couette flow: comparing wavelengths, and speeds in theory and experiment," Astrophys. J. 649, L145-147 (2006).

${ }^{28}$ D. Schmittt, T. Alboussière, D. Brito, P. Cardin, N. Gagnière, D. Jault, and H.-C. Nataf, "Rotating spherical Couette flow in a dipolar magnetic field: experimental study of magneto-inertial waves," J. Fluid Mech. 604, 175 (2008).

${ }^{29}$ P. Moresco and T. Alboussière, "Stability of Bödwaldt-Hartmann layer," Eur. J. Mech. B Fluids 23, 851 (2004).

${ }^{30}$ H. Araseki, I. R. Kirillov, G. V. Preslitsky, and A. P. Ogorodnikov, "Double-supply-frequency pressure pulsation in annular linear induction pump, part I: measurement and numerical analysis," Nucl. Eng. Design 195, 85 (2000).

${ }^{31}$ M. S. Paoletti and D. P. Lathrop, “Angular momentum transport in turbulent flow between independently rotating cylinders," Phys. Rev. Lett. 106, 024501 (2011).

${ }^{32}$ D. P. M. van Gils, S. G. Huisman, G. W. Bruggert, C. Sun, and D. Lohse, "Torque scaling in turbulent Taylor-Couette flow with co- and counterrotating cylinders," Phys. Rev. Lett. 106, 024502 (2011).

${ }^{33}$ B. Gallet, J. Herault, C. Laroche, F. Pétrélis, and S. Fauve, "Reversals of a large scale field generated over a turbulent background," Geophys. Astro. Fluid Dyn. (in press).

${ }^{34}$ Y. Zhao, O. Zikanov, and D. Krasnov, "Instability of magnetohydrodynamic flow in an annular channel at high Hartmann number," Phys. Fluids 23, 084103 (2011). 